\title{
Heavy quark mass effects on the virtual photon structure in QCD
}

\section{Yoshio Kitadono}

Dept. of Physics, Faculty of Science, Hiroshima University

Higashi Hiroshima 739-8526, Japan

E-mail: kitadono@theo.phys.sci.hiroshima-u.ac.jp

\section{Ken Sasaki}

Dept. of Physics, Faculty of Engineering, Yokohama National University

Yokohama 240-8501, Japan

E-mail: sasaki@ynu.ac.jp

\section{Takahiro Ueda}

Graduate School of Pure and Applied Sciences, University of Tsukuba

Tsukuba, Ibaraki 305-8571, Japan

E-mail: tueda@het.ph.tsukuba.ac.jp

\section{Tsuneo Uematsu*}

Dept. of Physics, Graduate School of Science, Kyoto University

Yoshida, Kyoto 606-8501, Japan

E-mail: uematsuescphys.kyoto-u.ac.jp

\begin{abstract}
We investigate the heavy quark mass effects on the virtual photon structure to the next-to-leading order (NLO) in QCD. Our formalism is based on the parton picture as well as on the operator product expansion in the framework of the mass-independent renormalization group equation. We evaluate the various components of the parton distribution functions inside the virtual photon in the presence of the heavy quark mass effects.
\end{abstract}

RADCOR 2009 - 9th International Symposium on Radiative Corrections (Applications of Quantum Field Theory to Phenomenology)

October 25-30 2009

Ascona, Switzerland

\footnotetext{
* Speaker.
} 


\section{Introduction}

In this talk we would like to address the question about the heavy quark mass effects on the virtual photon structure in QCD. The virtual photon structure is interesting, because it provides a good probe to study QCD dynamics in perturbation theory. Experimentally in the future linear collider (ILC), two-photon-process can be explored in the new kinematical region.

So far we have studied the unpolarized photon structure functions $F_{2}^{\gamma}$ and $F_{L}^{\gamma}$ to the next-tonext-to-leading order (NNLO) in QCD [1] and also the parton distribution functions to NNLO [2]. But there we have treated all the quarks are massless, and in that sense it does not describe the real situation. So here we would like to discuss about the heavy quark mass effects on the virtual photon structure, especially the parton distributions.

The photon structure can be studied in the two-photon processes in the $e^{+} e^{-}$collision which dominate over one-photon annihilation processes at high energies. Namely we consider the twophoton processes with double-tagging experiments where both of the outgoing $e^{+}$and $e^{-}$are detected. In particular we study the kinematical region in which one of the photons, which we call the 'probe photon', with the mass squared $-q^{2}=Q^{2}$, is much heavier than the other one, 'target photon', with the mass squared $-p^{2}=P^{2}$, which is in turn much larger than $\Lambda_{\mathrm{QCD}}^{2}$, i.e. $\Lambda_{\mathrm{QCD}}^{2} \ll P^{2} \ll Q^{2}$. In this kinematical region we can study the whole structure functions and the parton distributions, their shapes and magnitudes.

In the case of nucleon target the heavy quarks are treated as radiatively generated from the gluon and light quarks. For the virtual photon target, the heavy quarks are treated in the same way as the light quarks, namely both heavy and light quarks are radiatively generated from the photon target. We study heavy quark mass effects in the framework of mass-independent renormalization group method. Heavy quark mass effects for the real photon case were studied in the literature [3, 4, 5, 6. In our case of the virtual photon target we treat the heavy quarks on the same footing as the light quarks, except for the mass, as discussed below.

\section{Evolution equations}

Let us consider the case where we have $n_{f}-1$ massless quarks and one heavy quark $q^{n_{f}}=q_{H}^{\gamma}$. Of course it is straightforward to extend the present argument to the case with two or more heavy quarks. The DGLAP evolution equation reads [7]:

$$
\frac{d}{d \ln Q^{2}} \vec{q}^{\gamma}\left(x, Q^{2}, P^{2}\right)=\int_{x}^{1} \frac{d y}{y} \vec{q}^{\gamma}\left(y, Q^{2}, P^{2}\right) \hat{P}\left(\frac{x}{y}, Q^{2}\right)+\vec{k}\left(x, Q^{2}\right),
$$

where the parton distributions are denoted as a row vector $\vec{q}^{\gamma}\left(x, Q^{2}, P^{2}\right)=\left(q_{L S}^{\gamma}, q_{H}^{\gamma}, G^{\gamma}, q_{L N S}^{\gamma}\right)$, in which $G^{\gamma}\left(q_{H}^{\gamma}\right)$ denotes the gluon (heavy quark) distribution, while the flavor-singlet combination for the light-flavors, $q_{L S}^{\gamma}$, and the non-singlet combination $q_{L N S}^{\gamma}$ are defined as

$$
q_{L S}^{\gamma} \equiv \sum_{i=1}^{n_{f}-1} q_{L}^{i}, \quad q_{L N S}^{\gamma} \equiv \sum_{i=1}^{n_{f}-1} e_{i}^{2}\left(q_{L}^{i}-\frac{1}{n_{f}-1} q_{L S}^{\gamma}\right) .
$$

In the above equation $\hat{P}$ is the $4 \times 4$ matrix of the splitting function which consists of the $3 \times 3$ singlet sector of the $L S, H, G$ components and non-singlet sector of the $L N S$ component. 
Note that the moments of the splitting functions $P_{i j}$ are related to the anomalous dimensions of the quark, gluon and photon operators $\vec{O}_{n}, \gamma_{n}(g, \alpha)_{i j}$, and the coefficient function $C_{n}^{i}$ satisfies the following mass-independent renormalization group equation (RGE):

$$
\left[\mu \frac{\partial}{\partial \mu}+\beta(g) \frac{\partial}{\partial g}+\gamma_{m}(g) m \frac{\partial}{\partial m}-\gamma_{n}(g, \alpha)\right]_{i j} C_{n}^{j}\left(\frac{Q^{2}}{\mu^{2}}, \frac{m^{2}}{\mu^{2}}, \bar{g}\left(\mu^{2}\right), \alpha\right)=0,
$$

where $\gamma_{m}(g)$ is the anomalous dimension for the mass operator.

Now let us consider the $F_{2}^{\gamma}$ structure function which is given by the convolution of the parton distribution function $\vec{q}^{\gamma}$ and the coefficient function $\vec{C}_{n}$ :

$$
F_{2}^{\gamma}\left(x, Q^{2}, P^{2}\right)=\vec{q}^{\gamma}\left(y, Q^{2}, P^{2}, m^{2}\right) \otimes \vec{C}\left(\frac{x}{y}, \frac{\bar{m}^{2}}{Q^{2}}, \bar{g}\left(Q^{2}\right)\right),
$$

which represents the factorization property of the photon structure.

The moment of the parton distribution $\vec{q}^{\gamma}\left(y, Q^{2}, P^{2}, m^{2}\right)$ is given by

$$
\int_{0}^{1} d x x^{n-1} \vec{q}^{\gamma}\left(x, Q^{2}, P^{2}, m^{2}\right)=\vec{A}_{n}\left(1, \frac{\bar{m}^{2}\left(P^{2}\right)}{P^{2}}, \bar{g}\left(P^{2}\right)\right) T \exp \left[\int_{\bar{g}\left(Q^{2}\right)}^{\bar{g}\left(P^{2}\right)} d g \frac{\gamma_{n}(g, \alpha)}{\beta(g)}\right],
$$

where $\vec{A}_{n}$ is the photon matrix element of the operator $\vec{O}_{n}$. When the operator $\vec{O}_{n}$ is renormalized at $\mu^{2}$, the matrix element $\vec{A}_{n}$ is given as

$$
\left\langle\gamma\left(P^{2}\right)\left|\vec{O}_{n}\left(\mu^{2}\right)\right| \gamma\left(P^{2}\right)\right\rangle=\vec{A}_{n}\left(\frac{P^{2}}{\mu^{2}}, \frac{\bar{m}^{2}\left(\mu^{2}\right)}{\mu^{2}}, \bar{g}\left(\mu^{2}\right)\right),
$$

and remarkably it is perturbatively calculable in contrast to the real photon case. We should note that the mass dependence only resides in the $\left\langle\gamma\left|\vec{O}_{n}\right| \gamma\right\rangle$ and $\vec{C}_{n}$ from the above argument.

\section{Heavy quark effects on structure functions and parton distributions}

Our master formula for the structure function is given by

$$
\begin{aligned}
& M_{2}^{\gamma}\left(n, Q^{2}, P^{2}\right)=\int_{0}^{1} d x x^{n-2} F_{2}^{\gamma}\left(x, Q^{2}, P^{2}\right) \\
& =\frac{\alpha}{4 \pi} \frac{1}{2 \beta_{0}}\left[\frac{4 \pi}{\alpha_{s}\left(Q^{2}\right)} \sum_{i} \mathscr{L}_{i}^{n}\left(1-r^{d_{i}^{n}+1}\right)+\sum_{i} \mathscr{A}_{i}^{n}\left(1-r^{d_{i}^{n}}\right)+\sum_{i} \mathscr{B}_{i}^{n}\left(1-r^{d_{i}^{n}+1}\right)+\mathscr{C}^{n}\right]+\mathscr{O}\left(\alpha_{s}\right),
\end{aligned}
$$

where $r \equiv \alpha_{s}\left(Q^{2}\right) / \alpha_{s}\left(P^{2}\right), d_{i}^{n}=\lambda_{i}^{n} / 2 \beta_{0}$ with $\lambda_{i}^{n}(i= \pm, N S)$ being the eigenvalues of 1-loop anomalous dimensions. $\mathscr{L}_{i}^{n}, \mathscr{A}_{i}^{n}, \mathscr{B}_{i}^{n}$ and $\mathscr{C}^{n}$ are the coefficients determined by 1 and 2-loop anomalous dimensions and 1-loop coefficient functions [7].

In the presence of quark mass effects, the structure function can be decomposed as follows

$$
F_{2}^{\gamma}\left(x, Q^{2}, P^{2}\right)=\left.F_{2}^{\gamma}\left(x, Q^{2}, P^{2}\right)\right|_{\text {massless }}+\Delta F_{2}^{\gamma}\left(x, Q^{2}, P^{2}\right) .
$$

Now we note that the deviation of moment of the structure function to NLO in the massive quark limit: $P^{2} \ll m^{2}$ is given in terms of those for the parton distributions

$$
\Delta M_{2}^{\gamma}(n)=\left\langle e^{2}\right\rangle_{L} \Delta q_{L S}^{\gamma}(n)+e_{H}^{2} \Delta q_{H}^{\gamma}(n), \quad\left\langle e^{2}\right\rangle_{L} \equiv \sum_{i-1}^{n_{f}-1} e_{i}^{2} /\left(n_{f}-1\right),
$$




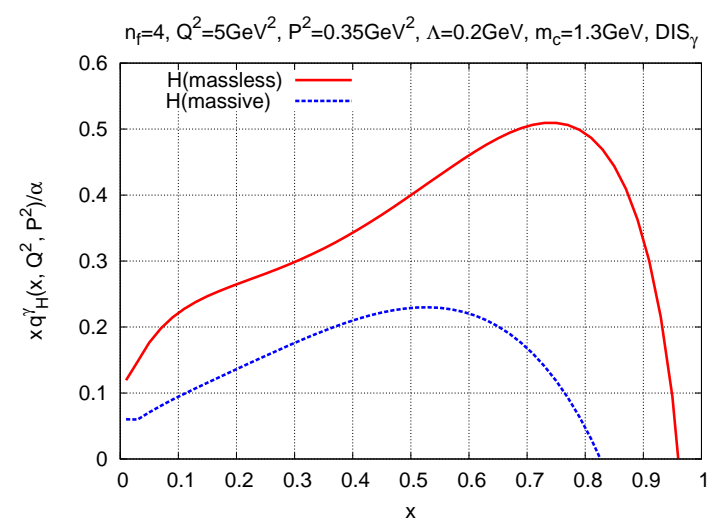

(a)

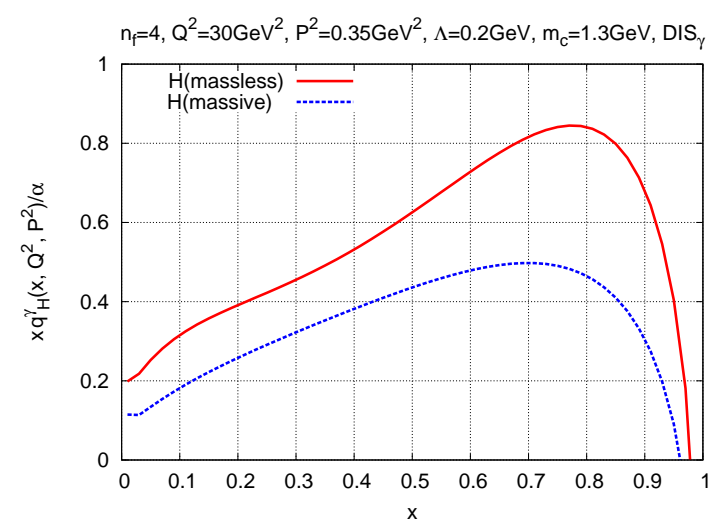

(b)

Figure 1: $x q_{H}^{\gamma}\left(x, Q^{2}, P^{2}\right)$ in DIS $\gamma$ scheme to NLO in QCD for (a) $Q^{2}=5 \mathrm{GeV}^{2}$ and (b) $Q^{2}=30 \mathrm{GeV}^{2}$ with $n_{f}=4, P^{2}=0.35 \mathrm{GeV}^{2}$ and $\Lambda=0.2 \mathrm{GeV}$, for the massless (red curve) and massive (blue curve) $c$ quark case.

where the deviation of the light-singlet and heavy quark distributions turns out to be

$$
\begin{aligned}
\Delta q_{L S}^{\gamma}(n) / \frac{\alpha}{8 \pi \beta_{0}}= & 2 \beta_{0} \frac{n_{f}-1}{n_{f}} \Delta \tilde{A}_{H}^{n}\left(1-r^{d_{N S}^{n}}\right)-\frac{n_{f}-1}{n_{f}} 2 \beta_{0} \frac{\gamma_{\psi \psi}^{(0), n}-\lambda_{-}^{n}}{\lambda_{+}^{n}-\lambda_{-}^{n}} \Delta \tilde{A}_{H}^{n}\left(1-r^{d_{+}^{n}}\right) \\
& -\frac{n_{f}-1}{n_{f}} 2 \beta_{0} \frac{\gamma_{\psi \psi}^{(0), n}-\lambda_{+}^{n}}{\lambda_{-}^{n}-\lambda_{+}^{n}} \Delta \tilde{A}_{H}^{n}\left(1-r^{d_{-}^{n}}\right), \\
\Delta q_{H}^{\gamma}(n) / \frac{\alpha}{8 \pi \beta_{0}}= & -2 \beta_{0} \frac{n_{f}-1}{n_{f}} \Delta \tilde{A}_{H}^{n}\left(1-r^{d_{N S}^{n}}\right)-\frac{1}{n_{f}} 2 \beta_{0} \frac{\gamma_{\psi \psi}^{(0), n}-\lambda_{-}^{n}}{\lambda_{+}^{n}-\lambda_{-}^{n}} \Delta \tilde{A}_{H}^{n}\left(1-r^{d_{+}^{n}}\right) \\
& -\frac{1}{n_{f}} 2 \beta_{0} \frac{\gamma_{\psi \psi}^{(0), n}-\lambda_{+}^{n}}{\lambda_{-}^{n}-\lambda_{+}^{n}} \Delta \tilde{A}_{H}^{n}\left(1-r^{d_{-}^{n}}\right)+2 \beta_{0} \Delta \tilde{A}_{H}^{n},
\end{aligned}
$$

while the deviation of the gluon and the light non-singlet quark distributions are given by

$$
\Delta G^{\gamma}(n) / \frac{\alpha}{8 \pi \beta_{0}}=2 \beta_{0} \Delta \tilde{A}_{H}^{n}\left\{\frac{\gamma_{G \psi}^{0, n}}{\lambda_{+}^{n}-\lambda_{-}^{n}}\left(1-r^{d_{+}^{n}}\right)+\frac{\gamma_{G \psi}^{0, n}}{\lambda_{-}^{n}-\lambda_{+}^{n}}\left(1-r^{d_{-}^{n}}\right)\right\}, \Delta q_{L N S}^{\gamma}(n)=0 .
$$

In the above equation, $\Delta \tilde{A}_{H}^{n}$ is related to the deviation of the operator matrix element due to heavy quark mass effects

$$
\left.\Delta \vec{A}_{n}\right|_{\mu^{2}=P^{2}}=\frac{\alpha}{4 \pi}\left(0, \Delta \tilde{A}_{H}^{n}, 0,0\right) .
$$

In the parton language, this is equivalent to take the initial condition for the heavy quark distribution to be different from those for the light-quark distributions. In the limit: $\Lambda_{\mathrm{QCD}}^{2} \ll P^{2} \ll m^{2} \ll Q^{2}$, $\Delta \tilde{A}_{H}^{n}$ is given by

$$
\Delta \tilde{A}_{H}^{n}=12 e_{H}^{2}\left[-\frac{n^{2}+n+2}{n(n+1)(n+2)}\left(\ln \frac{m^{2}}{P^{2}}+\sum_{j=1}^{n} \frac{1}{j}\right)+\frac{1}{n}-\frac{1}{n^{2}}+\frac{4}{(n+1)^{2}}-\frac{4}{(n+2)^{2}}\right] .
$$

Note that in the above heavy quark mass limit, we have no deviation for the coefficient functions to NLO in QCD [7]. 


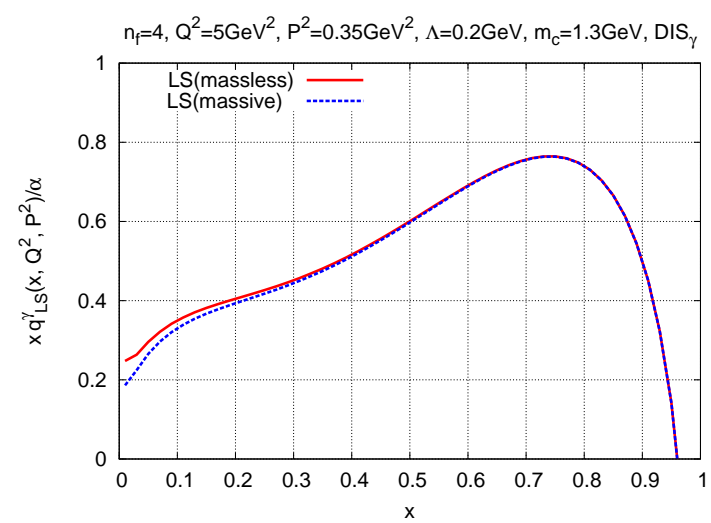

(a)

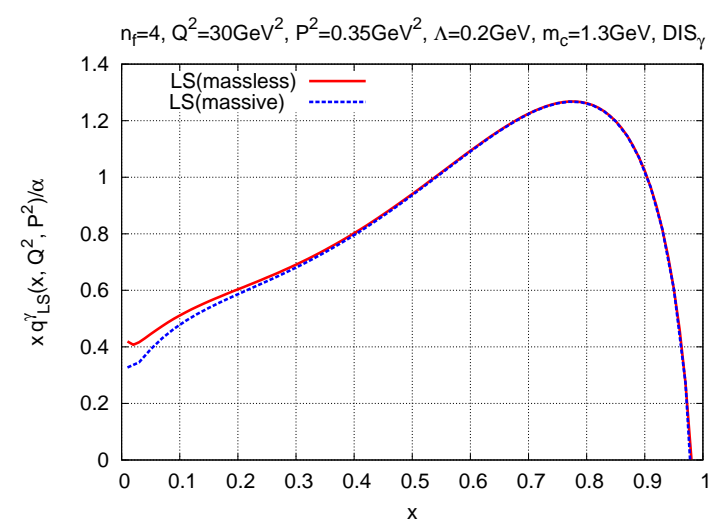

(b)

Figure 2: $x q_{L S}^{\gamma}\left(x, Q^{2}, P^{2}\right)$ in DIS $\gamma$ scheme to NLO in QCD for (a) $Q^{2}=5 \mathrm{GeV}^{2}$ and (b) $Q^{2}=30 \mathrm{GeV}^{2}$ with $n_{f}=4, P^{2}=0.35 \mathrm{GeV}^{2}$ and $\Lambda=0.2 \mathrm{GeV}$, for the massless (red curve) and massive (blue curve) $c$ quark case.

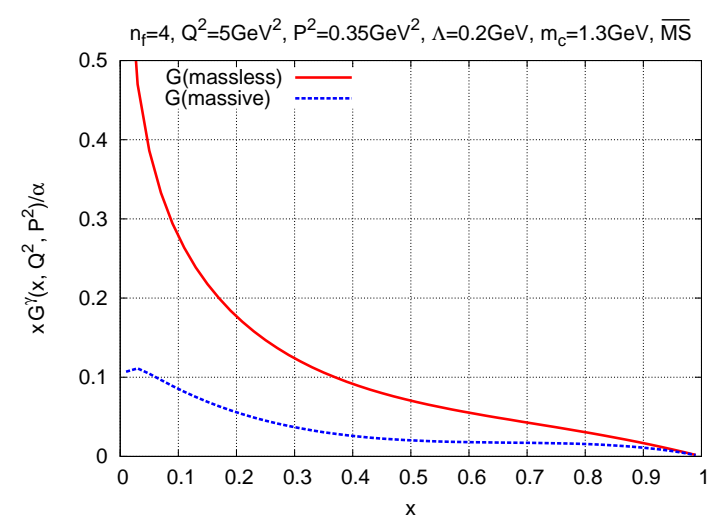

(a)

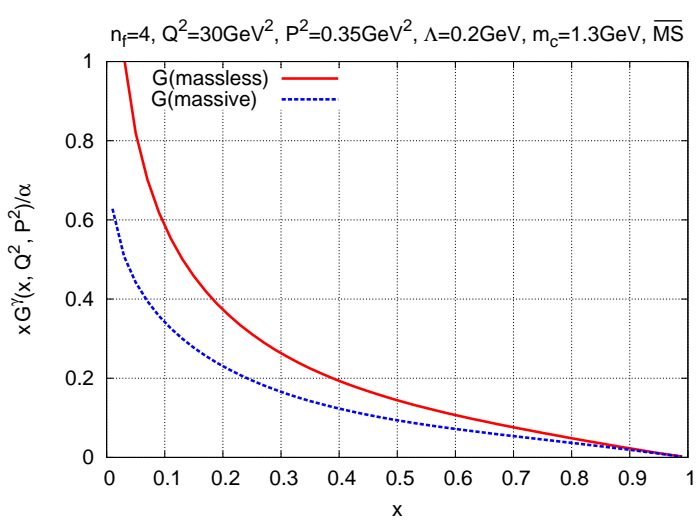

(b)

Figure 3: $x G^{\gamma}\left(x, Q^{2}, P^{2}\right)$ in $\overline{\mathrm{MS}}$ (DIS $\gamma$ ) scheme to NLO in QCD for (a) $Q^{2}=5 \mathrm{GeV}^{2}$ and (b) $Q^{2}=30 \mathrm{GeV}^{2}$ with $n_{f}=4, P^{2}=0.35 \mathrm{GeV}^{2}$ and $\Lambda=0.2 \mathrm{GeV}$, for the massless (red curve) and massive (blue curve) $c$ quark case.

\section{Numerical analysis}

By performing the inverse Mellin transform, we numerically evaluate the parton distributions for the case $n_{f}=4, P^{2}=0.35 \mathrm{GeV}^{2}, \Lambda=0.2 \mathrm{GeV}, Q^{2}=5$ and $30 \mathrm{GeV}^{2}$. We take $c$ quark to be a heavy quark with $m_{c}=1.3 \mathrm{GeV}$ as an input and assume that the other quarks, $u, d$ and $s$ are light-quarks and taken to be massless. We have studied the parton distribution functions in both $\overline{\mathrm{MS}}$ and DIS ${ }_{\gamma}$ schemes. In Figure 1 we have shown heavy quark distribution functions in DIS scheme $_{\gamma}$ for (a) $Q^{2}=5 \mathrm{GeV}^{2}$ and (b) $Q^{2}=30 \mathrm{GeV}^{2}$. The values of $Q^{2}$ and $P^{2}$ for the case (a) correspond to those of the PLUTO experiment [8], in which the effective virtual photon structure function was measured. Similarly the light-singlet quark and gluon distributions are shown in Figures 2 and 3. respectively. Note that for the gluon distribution, there is no difference between $\overline{\mathrm{MS}}$ and DIS $\gamma$ schemes at this order. In order to see the heavy quark effects, we plot, in addition, the each parton distribution which are obtained when $c$-quark is also set to be massless. Actually we can recover 
the massless case by setting $\Delta \tilde{A}_{H}^{n} \rightarrow 0$ in the above equation.

The heavy quark mass effects tend to reduce the values of parton distribution functions for the light-singlet, the heavy-quark and the gluon distributions except for the light nonsinglet distribution. These results could be explained by the suppression of the evolution range due to the mass of the heavy quark.

\section{Summary and outlook}

Based on DGLAP equation as well as on the OPE formalism we have studied the heavy quark mass effects on the parton (light singlet, heavy quark, gluon, light nonsinglet) distribution functions in the virtual photon up to the NLO in perturbative QCD. The heavy quark effect is included through the operator matrix element for heavy quark operator and is evaluated by the heavy quark mass limit $\left(\Lambda^{2} \ll P^{2} \ll m^{2}\right)$. In the language of the parton picture, the heavy quark effects are arising from the initial condition for the heavy quark distribution.

We have not taken into account the kinematical threshold effects which manifest as the presence of the maximal values of the Bjorken variable. We need some improvement in which the threshold effects are included. We should also investigate the general kinematical region where $P^{2}$ and $m^{2}$ are of the same order. We also note that the general-mass variable-flavor-number scheme (GM-VFNS), which has now become a popular framework for the global analyses of parton distributions, should be implemented in the present analysis which is under investigation.

\section{Acknowledgements}

I would like to thank Thomas Gehrmann and the other organizers of RADCOR 2009 for their hospitality at this very productive symposium.

\section{References}

[1] T. Ueda, K. Sasaki and T. Uematsu, Phys. Rev. D75 (2007) 114009 [hep-ph/ 0703296 ].

[2] T. Ueda, K. Sasaki and T. Uematsu, Eur. Phys. J. C62 (2009) 467 [hep-ph/ 0902 . 3086]; POS (RADCOR 2007)036.

[3] M. Glück and E. Reya, Phys. Rev. D28 (1983) 2749; M. Glück and E. Reya and A. Vogt, Phys. Rev. D46 (1992) 1973; M. Glück, E. Reya and C. Sieg, Phys. Lett. B503 (2001) 285; Eur. Phys J. C20 (2001) 271; M .Glück, E. Reya and I.Schienbein, Phys. Rev. D60 (1999) 054019; Phys. Rev. D63 (2001) 074008; I. Schienbein, Annals of Physics 301 (2002) 128.

[4] K. Sasaki, J. Soffer and T. Uematsu, Phys. Rev. D66 (2002) 034014 [hep-ph/ 0205159 ].

[5] F. Cornet, P. Jankowski, M. Krawczyk and A. Lorca, Phys. Rev. D68 (2003) 014010 [hep-ph/0212160].

[6] F. Cornet, P. Jankowski and M. Krawczyk, Phys. Rev. D70 (2004) 093004 [hep-ph / 0404063 ].

[7] Y. Kitadono, K. Sasaki, T. Ueda and T. Uematsu, Prog. Theor. Phys. 121 (2009) 495 [hep-ph/0812 .1083]; arXiv:0912.4829[hep-ph].

[8] Ch. Berger et al. (PLUTO Collaboration), Phys. Lett. B142 (1984) 119. 